\title{
Research Article \\ Compact Spacelike Hypersurfaces with Constant Mean Curvature in the Antide Sitter Space
}

\author{
Henrique F. de Lima and Joseilson R. de Lima \\ Departamento de Matemática e Estatística, Universidade Federal de Campina Grande, Campina Grande, \\ 58109-970, Paraíba, Brazil
}

Correspondence should be addressed to Henrique F. de Lima, henrique@dme.ufcg.edu.br

Received 8 July 2009; Accepted 6 October 2009

Recommended by Christian Corda

We obtain a height estimate concerning to a compact spacelike hypersurface $\Sigma^{n}$ immersed with constant mean curvature $H$ in the anti-de Sitter space $\mathbb{H}_{1}^{n+1}$, when its boundary $\partial \Sigma$ is contained into an umbilical spacelike hypersurface of this spacetime which is isometric to the hyperbolic space $\mathbb{H}^{n}$. Our estimate depends only on the value of $H$ and on the geometry of $\partial \Sigma$. As applications of our estimate, we obtain a characterization of hyperbolic domains of $\mathbb{H}_{1}^{n+1}$ and nonexistence results in connection with such types of hypersurfaces.

Copyright (C) 2009 H. F. de Lima and J. R. de Lima. This is an open access article distributed under the Creative Commons Attribution License, which permits unrestricted use, distribution, and reproduction in any medium, provided the original work is properly cited.

\section{Introduction}

Interest in the study of spacelike hypersurfaces in Lorentzian manifolds has increased very much in recent years, from both the physical and mathematical points of view. For example, it was pointed out by J. Marsden and F. Tipler in [1] and S. Stumbles in [2] that spacelike hypersurfaces with constant mean curvature in arbitrary spacetimes play an important part in the relativity theory. They are convenient as initial hypersurfaces for the Cauchy problem in arbitrary spacetime and for studying the propagation of gravitational radiation. From a mathematical point of view, that interest is also motivated by the fact that these hypersurfaces exhibit nice Bernstein-type properties. Actually, E. Calabi in [3], for $n \leq 4$, and Cheng and Yau in [4], for arbitrary $n$, showed that the only complete immersed spacelike hypersurfaces of the $(n+1)$-dimensional Lorentz-Minkowski space $\mathbb{L}^{n+1}$ with zero mean curvature are the spacelike hyperplanes.

Related with the compact case, Alías and Malacarne in [5] showed that the only compact spacelike hypersurfaces having constant higher-order mean curvature and spherical boundary in $\mathbb{L}^{n+1}$ are the hyperplanar balls with zero higher-order mean curvature, and the hyperbolic caps with nonzero constant higher-order mean curvature (cf. [6] for the case of constant mean curvature and [7] for the case of constant scalar curvature; see also [8] 
for the case of 2-dimensional surfaces in $\mathbb{L}^{3}$ ). Also considering the compact case, R. López obtained a sharp estimate for the height of compact spacelike surfaces $\Sigma^{2}$ immersed into the 3-dimensional Lorentz-Minkowski space $\mathbb{L}^{3}$ with constant mean curvature (cf. [9], Theorem 1). For the case of constant higher-order mean curvature, by applying the techniques used by D. Hoffman et al. in [10], the first author obtained another sharp height estimate for compact spacelike hypersurfaces immersed in the $(n+1)$-dimensional Lorentz-Minkowski space $\mathbb{L}^{n+1}$ (cf. [11], Theorem 4.2).

Also recently, the first author obtained some geometric estimates concerning to a spacelike hypersurface immersed with some constant higher-order mean curvature in de Sitter space (cf. [12]), also, in a Lorentzian product space with Colares (cf. [13]) and in a conformally stationary Lorentz manifold with A. Caminha (cf. [14]). We note that, in each one of these papers, the authors have used their geometric estimates to study the existence of certain types of spacelike hypersurfaces in such spacetimes.

In [15] the first author and A. Caminha have studied complete vertical graphs of constant mean curvature in the hyperbolic and steady state spaces. Under appropriate restrictions on the values of the mean curvature and the growth of the height function, they obtained necessary conditions for the existence of such a graph. In the 2-dimensional case they applied their analytical framework to prove Bernstein-type results in each of these ambient spaces.

We note that Albujer and Alías have also recently considered in [16] complete spacelike hypersurfaces with constant mean curvature in the steady state space. They proved that if the hypersurface is bounded away from the infinity of the ambient space, then the mean curvature must be $H=1$. In the 2-dimensional case they concluded that the only complete spacelike surfaces with constant mean curvature which are bounded away from the infinity are the totally umbilical flat surfaces. Moreover, considering the generalized Robertson-Walker spacetime model of the steady state space, they extended their results to a wider family of spacetimes.

In this paper we deal with a compact spacelike hypersurface $\Sigma^{n}$ immersed with constant mean curvature $H$ in the antide Sitter space $\mathbb{H}_{1}^{n+1}$, which is a particular model of Robertson-Walker spacetime given by $\mathbb{H}_{1}^{n+1}=-(-\pi / 2, \pi / 2) \times_{\cos t} \mathbb{H}^{n}$, where $\mathbb{H}^{n}$ denotes the $n$-dimensional hyperbolic space (cf. Section 3 ). In this setting, by supposing its boundary $\partial \Sigma$ contained into some slice of $\mathbb{H}_{1}^{n+1}$, we obtain an estimate for its vertical height function $h$. We prove the following result (cf. Theorem 3.2):

Let $\psi: \Sigma^{n} \rightarrow \mathbb{H}_{1}^{n+1}$ be a compact spacelike hypersurface whose boundary $\partial \Sigma$ is contained in some slice $\mathbb{H}_{t_{0}}^{n}=\left\{t_{0}\right\} \times \mathbb{H}^{n}$. Suppose that the mean curvature $H>1$ is constant.

(i) If $-\pi / 2<t_{0} \leq 0$ and $\Sigma^{n}$ is contained into the chronological past with respect to $\mathbb{H}_{t_{0}}^{n}$ then the height $h$ of $\Sigma^{n}$ satisfies

$$
t_{0} \geq h \geq t_{0}-\frac{1}{H-1}\left(C \cos t_{0}-\cos h\right) .
$$

(ii) If $0 \leq t_{0}<\pi / 2$ and $\Sigma^{n}$ is contained into the chronological future with respect to $\mathbb{H}_{t_{0}}^{n}$, then the height $h$ of $\Sigma^{n}$ satisfies

$$
t_{0} \leq h \leq t_{0}+\frac{1}{H-1}\left(C \cos t_{0}-\cos h\right) .
$$

Here $C=\max _{\partial \Sigma}(\cosh \theta)$ and $\theta$ is the hyperbolic angle between the Gauss map $N$ of $\Sigma$ and $\partial_{t}$. 
Suitable formulae for the Laplacians of the height function and a support-like function naturally attached to a spacelike hypersurface $\Sigma^{n}$ immersed in $\mathbb{H}_{1}^{n+1}$ constitute the analytical tools that we use to get our estimate (cf. Lemma 2.1).

It is important to point out that our estimate depends only on the value of the mean curvature and on the geometry of the boundary of the hypersurface. On the other hand, we recall that an integral curve of the unit time-like vector field $\partial_{t}$ is called a comoving observer and, when $p$ is a point of a spacelike hypersurface $\Sigma^{n}$ immersed into a Robertson-Walker spacetime $-\mathbb{R} \times{ }_{f} M^{n}, \partial_{t}(p)$ is called an instantaneous comoving observer. In this setting, among the instantaneous observers at $p, \partial_{t}(p)$ and $N(p)$ appear naturally. From the orthogonal decomposition $N(p)=-\left\langle N, \partial_{t}\right\rangle_{p} \partial_{t}(p)+\left(\pi_{M}\right)_{*} N(p)$ where $\pi_{M}$ denotes the canonical projection from $-\mathbb{R} \times_{f} M^{n}$ onto the Riemannian fiber $M^{n}$, we have that $\cosh \theta(p)$ corresponds to the energy $e(p)$ that $\partial_{t}(p)$ measures for the normal observer $N(p)$. Furthermore, the speed $|v(p)|$ of the Newtonian velocity $v(p):=(1 / e(p))\left(\pi_{M}\right)_{*} N(p)$ that $\partial_{t}(p)$ measures for $N(p)$ satisfies the equation $|v(p)|^{2}=\tanh \theta(p)$. So, a physical consequence of the boundedness of the hyperbolic angle $\theta$ between the Gauss map $N$ of the spacelike hypersurface $\Sigma^{n}$ and $\partial_{t}$ is that the speed of the Newtonian velocity that the instantaneous comoving observer measures for the normal observer does not approach the speed of light 1 on $\Sigma^{n}$ (see [17], Sections 2.1 and 3.1, and [18]; see also [19], Chapter 12).

As an application of our height estimate, we obtain an characterization of hyperbolic domains of $\mathbb{H}_{1}^{n+1}$ (cf. Corollary 4.3). Furthermore, we establish nonexistence results in connection with such types of hypersurfaces (cf. Corollaries 4.4 and 4.5). For example, we prove the following.

There is no compact spacelike hypersurface $\psi: \Sigma^{n} \rightarrow \mathbb{H}_{1}^{n+1}$ with constant mean curvature $H \geq 2$ and tangent to the slice $\mathbb{H}_{0}^{n}$ along its boundary.

Finally, we observe that an interesting feature of the four-dimensional antide Sitter space $\mathbb{H}_{1}^{4}$ is that, as a cosmological model, this spacetime is a maximally symmetric universe with constant negative curvature, which is conformally related to half of the Einstein static universe. Consequently, $\mathbb{H}_{1}^{4}$ represents a (locally) unique solution to Einstein's equation in the absence of any ordinary matter or gravitational radiation. In this setting, this spacetime may be thought of as a ground state of general relativity (cf. [20], Chapter 8; see also [21], Chapter 6, and [22], Chapter 14).

\section{Preliminaries}

In what follows, if $\bar{M}^{n+1}$ is a connected semi-Riemannian manifold with metric $\bar{g}=\langle$,$\rangle ,$ we let $\Phi(\bar{M})$ denote the ring of smooth functions $\phi: \bar{M}^{n+1} \rightarrow \mathbb{R}$ and $\mathfrak{X}(\bar{M})$ the algebra of smooth vector fields on $\bar{M}^{n+1}$. We also write $\bar{\nabla}$ for the Levi-Civita connection of $\bar{M}^{n+1}$.

Let $M^{n}$ be a connected, $n$-dimensional $(n \geq 2)$ oriented Riemannian manifold, $I$ a 1 dimensional manifold (either a circle or an open interval of $\mathbb{R}$ ), and $f: I \rightarrow \mathbb{R}$ a positive smooth function. In the product differentiable manifold $\bar{M}^{n+1}=I \times M^{n}$, let $\pi_{I}$ and $\pi_{M}$ denote the projections onto the factors $I$ and $M^{n}$, respectively.

A particular class of Lorentzian manifolds (spacetimes) is the one obtained by furnishing $\bar{M}^{n+1}$ with the metric

$$
\langle v, w\rangle_{p}=-\left\langle\left(\pi_{I}\right)_{*} v,\left(\pi_{I}\right)_{*} w\right\rangle+\left(f \circ \pi_{I}\right)(p)^{2}\left\langle\left(\pi_{M}\right)_{*} v,\left(\pi_{M}\right)_{*} w\right\rangle
$$


for all $p \in \bar{M}^{n+1}$ and all $v, w \in T_{p} \bar{M}$. Such a space is called (following the terminology introduced in [23]) a Generalized Robertson-Walker (GRW) spacetime, and in what follows we shall write $\bar{M}^{n+1}=-I \times_{f} M^{n}$ to denote it. In particular, when $M^{n}$ has constant sectional curvature, then $-I \times_{f} M^{n}$ is classically called a Robertson-Walker (RW) spacetime (cf. [19]). It is not difficult to see that a GRW spacetime $-I \times_{f} M^{n}$ has constant sectional curvature $\overline{\mathcal{\kappa}}$ if, and only if, the Riemannian fiber $M^{n}$ has constant sectional curvature $\kappa$ (i.e., $-I \times_{f} M^{n}$ is in fact a RW spacetime) and the warping function $f$ satisfies the following differential equations:

$$
\frac{f^{\prime \prime}}{f}=\bar{\kappa}=\frac{\left(f^{\prime}\right)^{2}+\kappa}{f^{2}}
$$

(see, for instance, [24], Corollary 9.107).

We recall that a tangent vector field $K$ on a spacetime $\bar{M}^{n+1}$ is said to be conformal if the Lie derivative with respect to $K$ of the metric $\langle$,$\rangle of \bar{M}^{n+1}$ satisfies

$$
\mathcal{L}_{K}\langle,\rangle=2 \phi\langle,\rangle
$$

for a certain smooth function $\phi \in \mathscr{\Phi}\left(\bar{M}^{n+1}\right)$. Since $\mathcal{L}_{K}(X)=[K, X]$ for all $X \in \mathfrak{X}(\bar{M})$, it follows from the tensorial character of $\mathcal{L}_{K}$ that $K \in \mathfrak{X}(\bar{M})$ is conformal if and only if

$$
\left\langle\bar{\nabla}_{X} K, Y\right\rangle+\left\langle X, \bar{\nabla}_{Y} K\right\rangle=2 \phi\langle X, Y\rangle
$$

for all $X, Y \in \mathfrak{X}(\bar{M})$. In particular, $K$ is a Killing vector field relatively to the metric $\langle$,$\rangle if and$ only if $\phi \equiv 0$.

We observe that when $\bar{M}^{n+1}=-I \times_{f} M^{n}$ is a GRW spacetime, the vector field

$$
K=f \partial_{t}=\left(f \circ \pi_{I}\right) \partial_{t}
$$

is conformal and closed (in the sense that its dual 1-form is closed), with conformal factor $\phi=f^{\prime}$, where the prime denotes differentiation with respect to $t \in I$ (cf. [25]).

A smooth immersion $\psi: \Sigma^{n} \rightarrow \bar{M}^{n+1}$ of an $n$-dimensional connected manifold $\Sigma^{n}$ is said to be a spacelike hypersurface if the induced metric via $\psi$ is a Riemannian metric on $\Sigma^{n}$, which, as usual, is also denoted for $\langle$,$\rangle . In that case, since$

$$
\partial_{t}=\left(\frac{\partial}{\partial_{t}}\right)_{(t, x)}, \quad(t, x) \in-I \times_{f} M^{n}
$$

is a unitary time-like vector field globally defined on the ambient GRW spacetime, then there exists a unique time-like unitary normal field $N$ globally defined on the spacelike hypersurface $\Sigma^{n}$ which is in the same time-orientation as $\partial_{t}$, so that

$$
\left\langle N, \partial_{t}\right\rangle \leq-1<0 \quad \text { on } \Sigma^{n}
$$


We will refer to that normal field $N$ as the future-pointing Gauss map of the spacelike hypersurface $\Sigma^{n}$. Its opposite will be referred as the past-pointing Gauss map of $\Sigma^{n}$.

In this setting, let $A: \mathfrak{X}(\Sigma) \rightarrow \mathfrak{X}(\Sigma)$ stand for the shape operator (or Weingarten endomorphism) of $\Sigma^{n}$ with respect to either the future or the past-pointing Gauss map N. It is well known that $A$ defines a self-adjoint linear operator on each tangent space $T_{p} \Sigma$, and its eigenvalues $\kappa_{1}(p), \ldots, \kappa_{n}(p)$ are the principal curvatures of $\Sigma^{n}$ at $p$. Associated to the shape operator $A$ there are $n$ algebraic invariants given by

$$
S_{r}(p)=\sigma_{r}\left(\kappa_{1}(p), \ldots, \kappa_{n}(p)\right), \quad 1 \leq r \leq n,
$$

where $\sigma_{r}: \mathbb{R}^{n} \rightarrow \mathbb{R}$ is the elementary symmetric function in $\mathbb{R}^{n}$ given by

$$
\sigma_{r}\left(x_{1}, \ldots, x_{n}\right)=\sum_{i_{1}<\cdots<i_{r}} x_{i_{1}} \cdots x_{i_{r}}
$$

Observe that the characteristic polynomial of $A$ can be written in terms of the $S_{r}^{\prime} s$ as

$$
\operatorname{det}(t I-A)=\sum_{r=0}^{n}(-1)^{r} S_{r} t^{n-r}
$$

where $S_{0}=1$ by definition. The $r$-mean curvature $H_{r}$ of the spacelike hypersurface $\Sigma^{n}$ is then defined by

$$
\left(\begin{array}{l}
n \\
r
\end{array}\right) H_{r}=(-1)^{r} S_{r}\left(\kappa_{1}, \ldots, \kappa_{n}\right)=S_{r}\left(-\kappa_{1}, \ldots,-\kappa_{n}\right) .
$$

In particular, when $r=1$,

$$
H_{1}=-\frac{1}{n} \sum_{i=1}^{n} \kappa_{i}=-\frac{1}{n} \operatorname{tr}(A)=H
$$

is the mean curvature of $\Sigma^{n}$, which is the main extrinsic curvature of the hypersurface. The choice of the sign $(-1)^{r}$ in our definition of $H_{r}$ is motivated by the fact that in that case the mean curvature vector is given by $\vec{H}=H N$. Therefore, $H(p)>0$ at a point $p \in \Sigma^{n}$ if and only if $\vec{H}(p)$ is in the same time-orientation as $N(p)$ (in the sense that $\langle\vec{H}, N\rangle_{p}<0$ ).

When $r=2, H_{2}$ defines a geometric quantity which is related to the (intrinsic) scalar curvature $R$ of the hypersurface. For instance, when the ambient spacetime $\bar{M}^{n+1}$ has constant sectional curvature $\bar{\kappa}$, it follows from the Gauss equation that

$$
R=n(n-1)\left(\bar{\kappa}-H_{2}\right) .
$$


Moreover, in the case of a 2-dimensional surface, denoting by $K_{\Sigma}$ the Gaussian curvature of the spacelike surface $\psi: \Sigma^{2} \rightarrow \bar{M}^{3}$, we have that

$$
K_{\Sigma}=\bar{\kappa}-H_{2}
$$

As before, let $\bar{M}^{n+1}=-I \times_{f} M^{n}$ be a GRW. For a fixed $t_{0} \in I$, we say that $M_{t_{0}}^{n}=\left\{t_{0}\right\} \times M^{n}$ is a slice of $\bar{M}^{n+1}$. It was proved by L.J. Alías et al. in [23] that each slice $M_{t_{0}}^{n}$ is an umbilical spacelike hypersurface with constant $r$-mean curvature, equal to $\left(f^{\prime}\left(t_{0}\right) / f\left(t_{0}\right)\right)^{r}$ with respect to $\partial_{t}$ (see also Example 5.6 in [26]). Whenever we talk about the mean curvature of the slices of a GRW, we shall assume that it is computed with respect to $\partial_{t}$. Also, if the (vertical) height function $h: \Sigma^{n} \rightarrow I$ of $\Sigma^{n}$, given by $h=\pi_{I} \circ \psi$, is such that $h \leq t_{0}\left(h \geq t_{0}\right)$ for some $t_{0} \in I$, then we say that $\Sigma^{n}$ is a spacelike hypersurface contained into the chronological past (chronological future) with respect to the slice $M_{t_{0}}^{n}$.

To close this section, we present the analytical framework that we will use to obtain our estimates. The formulae collected in the following lemma are particular cases of ones obtained by L.J. Alías and A.G. Colares (cf. [27], Lemma 4.1 and Corollary 8.4).

Lemma 2.1. Let $\psi: \Sigma^{n} \rightarrow-I \times{ }_{f} M^{n}$ be a spacelike hypersurface immersed into a GRW spacetime, with Gauss map $N$ and denote for $h=\pi_{I} \circ \psi$ the height function of $\Sigma^{n}$. Then

$$
\Delta h=-(\ln f)^{\prime}(h)\left(n+|\nabla h|^{2}\right)-n H\left\langle N, \partial_{t}\right\rangle
$$

Moreover, by supposing $\bar{M}^{n+1}$ an RW spacetime with Riemannian fiber $M^{n}$ of constant sectional curvature $\kappa$,

$$
\begin{aligned}
\Delta\left(f(h)\left\langle N, \partial_{t}\right\rangle\right)= & n f(h)\left\langle\nabla H, \partial_{t}\right\rangle+n H f^{\prime}(h) \\
& +n f(h)\left\langle N, \partial_{t}\right\rangle\left(n H^{2}-(n-1) H_{2}\right) \\
& +(n-1) f(h)\left\langle N, \partial_{t}\right\rangle\left(\frac{\kappa}{f^{2}(h)}-(\ln f)^{\prime \prime}(h)\right)|\nabla h|^{2} .
\end{aligned}
$$

Remark 2.2. For alternative proofs of the previous lemma, we suggest $[11,15,18,28]$.

\section{Height Estimate for Spacelike Hypersurfaces in $\mathbb{H}_{1}^{n+1}$}

In what follows we consider a particular model of RW spacetime, the antide Sitter space, namely

$$
\mathbb{H}_{1}^{n+1}=-\left(-\frac{\pi}{2}, \frac{\pi}{2}\right) \times \cos t \mathbb{H}^{n}
$$

where $\mathbb{H}^{n}$ denotes the $n$-dimensional hyperbolic space (see [29], Chapter 5). 
Remark 3.1. The spacetime $\mathbb{H}_{1}^{n+1}$ can also be regarded as the hyperquadric

$$
\mathbb{H}_{1}^{n+1}=\left\{p \in \mathbb{R}_{2}^{n+2} ;\langle p, p\rangle=-1\right\}
$$

in the indefinite index two flat space $\mathbb{R}_{2}^{n+2}$. For any timelike unit vector $a \in \mathbb{R}_{2}^{n+2}$, we have that the closed and conformal vector field $K$ given by

$$
K(p)=a+\langle a, p\rangle p, \quad p \in \mathbb{H}_{1}^{n+1}
$$

is timelike on the open set consisting of the points $p \in \mathbb{H}_{1}^{n+1}$ such that $\langle a, p\rangle^{2}<1$. This open set has two connect components and the distribution on $\mathbb{H}_{1}^{n+1}$ orthogonal to $K$ provides a foliation $\mathcal{F}(K)$ in this spacetime by means of the umbilical spacelike hypersurfaces $\langle p, a\rangle=\tau$, $-1<\tau<1$, which are isometric to two copies of hyperbolic spaces $\mathbb{H}^{n}$ with constant sectional curvature $-1 /\left(1+\tau^{2}\right)$. Consequently, each of these two components can be described as the Lorentzian warped product $-(-\pi / 2, \pi / 2) \times \cos t \mathbb{H}^{n}$ (see [25], Example 3 and Proposition 1).

Now, we present our main result.

Theorem 3.2. (Height Estimate) Let $\psi: \Sigma^{n} \rightarrow \mathbb{H}_{1}^{n+1}$ be a compact spacelike hypersurface whose boundary $\partial \Sigma$ is contained in some slice $\mathbb{H}_{t_{0}}^{n}=\left\{t_{0}\right\} \times \mathbb{H}^{n}$. Suppose that the mean curvature $H>1$ is constant.

(i) If $-\pi / 2<t_{0} \leq 0$ and $\Sigma^{n}$ is contained into the chronological past with respect to $\mathbb{H}_{t_{0}}^{n}$, then the height $h$ of $\Sigma^{n}$ satisfies

$$
t_{0} \geq h \geq t_{0}-\frac{1}{H-1}\left(C \cos t_{0}-\cos h\right)
$$

(ii) If $0 \leq t_{0}<\frac{\pi}{2}$ and $\Sigma^{n}$ is contained into the chronological future with respect to $\mathbb{H}_{t_{0}}^{n}$, then the height $h$ of $\Sigma^{n}$ satisfies

$$
t_{0} \leq h \leq t_{0}+\frac{1}{H-1}\left(C \cos t_{0}-\cos h\right)
$$

Here $C=\max _{\partial \Sigma}(\cosh \theta)$ and $\theta$ is the hyperbolic angle between the Gauss map $N$ of $\Sigma$ and $\partial_{t}$.

Proof. Suppose initially that $-\pi / 2<t_{0} \leq 0$ and $\Sigma^{n}$ is contained into the chronological past with respect to $\mathbb{H}_{t_{0}}^{n}$. From Lemma 2.1, we have

$$
\Delta h=\tan h\left(n+|\nabla h|^{2}\right)-n H\left\langle N, \partial_{t}\right\rangle
$$

Then, since $h \leq t_{0} \leq 0$, as a consequence of the maximum principle we must have $\Delta h(p) \geq 0$ for some point $p \in \Sigma^{n}$. Consequently, taking into account that we are supposing $H>1$, we conclude that the Gauss map $N$ of $\Sigma^{n}$ is future-pointing, that is,

$$
\left\langle N, \partial_{t}\right\rangle \leq-1
$$


on $\Sigma^{n}$. Now, in order to get our estimate, we define on $\Sigma^{n}$ the function

$$
\varphi=c\left(h-t_{0}\right)-\cos h\left\langle N, \partial_{t}\right\rangle
$$

where $c$ is a negative constant to be determined. By computing the Laplacian of $\varphi$ with the aid of Lemma 2.1, we get

$$
\begin{aligned}
\Delta \varphi= & C \tan h\left(n+|\nabla h|^{2}\right)-c n H\left\langle N, \partial_{t}\right\rangle+n H \sin h \\
& -\cos h\left\langle N, \partial_{t}\right\rangle\left(n^{2} H^{2}-n(n-1) H_{2}\right),
\end{aligned}
$$

where we have used the fact that the Riemannian fiber of $\mathbb{H}_{1}^{n+1}$ has constant sectional curvature $\kappa=-1$. Moreover, again as a consequence of the maximum principle, if $\Delta \varphi \geq 0$, then

$$
\begin{aligned}
\varphi & \leq\left.\varphi\right|_{\partial \Sigma}=\left(-\left.\left\langle N, \partial_{t}\right\rangle\right|_{\partial \Sigma}\right) \cos t_{0}=\left(\left.\cosh \theta\right|_{\partial \Sigma}\right) \cos t_{0} \\
& \leq \max _{\partial \Sigma}(\cosh \theta) \cos t_{0}=C \cos t_{0}
\end{aligned}
$$

on $\Sigma^{n}$, and

$$
0 \geq t_{0} \geq h \geq t_{0}+\frac{1}{c}\left(C \cos t_{0}-\cos h\right)
$$

We claim that it is possible to choose $c$ such that $\Delta \varphi \geq 0$. In fact, for all constant $c<0$, it yields

$$
C \tan h\left(n+|\nabla h|^{2}\right) \geq 0
$$

Putting this together with the Cauchy-Schwarz inequality $H^{2}-H_{2} \geq 0$ into the above expression of $\Delta \varphi$, we obtain

$$
\Delta \varphi \geq n H\left(-\left\langle N, \partial_{t}\right\rangle(H \cos h+c)-1\right)
$$

Thus, since the Gauss map $N$ of $\Sigma^{n}$ is future-pointing, by taking

$$
c=1-H
$$


we get that $\Delta \varphi \geq 0$. Therefore,

$$
t_{0} \geq h \geq t_{0}-\frac{1}{H-1}\left(C \cos t_{0}-\cos h\right)
$$

Now, suppose that $0 \leq t_{0}<\pi / 2$ and that $\Sigma$ is contained into the chronological future with respect to $\mathbb{H}_{t_{0}}^{n}$. In this case (again as a consequence of the maximum principle applied to the height function $h$ ), we have that Gauss map $N$ of $\Sigma^{n}$ is past-pointing, that is,

$$
\left\langle N, \partial_{t}\right\rangle \geq 1
$$

on $\Sigma^{n}$. Thus, we define on $\Sigma$ the function

$$
\varphi=c\left(h-t_{0}\right)+\cos h\left\langle N, \partial_{t}\right\rangle
$$

where $c$ is a positive constant to be determined. From this point, by taking

$$
c=H-1
$$

and working in a similar way as in the previous case we conclude that

$$
t_{0} \leq h \leq t_{0}+\frac{1}{H-1}\left(C \cos t_{0}-\cos h\right)
$$

Remark 3.3. Related to our previous theorem, it is important to observe the following facts.

(a) We note that, while in the Riemannian case (from the Cauchy-Schwarz inequality) the support function $\left\langle N, \partial_{t}\right\rangle$ of $\Sigma^{n}$ is always bounded, in the Lorentzian setting this boundedness occurs in a natural manner only when the spacelike hypersurface $\Sigma^{n}$ is compact. Consequently, in this last case, it is plausible that for an estimate of the vertical height $h$ must appear a term that depends on the geometry of the spacelike hypersurface. For example, the estimate of López for the height of a compact spacelike surface $\Sigma^{2}$ immersed with constant mean curvature into the 3-dimensional Lorentz-Minkowski space $\mathbb{L}^{3}$ and whose boundary $\partial \Sigma$ is included in a plane $\Pi$ depends on the value of the mean curvature and on the area of the region of $\Sigma^{2}$ above the plane $\Pi$ (cf. [9], Theorem 1). On the other hand, from Theorem 3.2, we see that our estimate depends on the value of the mean curvature and on the geometry of the boundary $\partial \Sigma$.

(b) Geometrically, observing that $\left|\left\langle N, \partial_{t}\right\rangle\right|=\cosh \theta$, we see that the boundedness of the hyperbolic angle $\theta$ means that (at each point $p \in \Sigma^{n}$ ) the normal direction $N(p)$ remains far from the light cone corresponding to $\partial_{t}(p)$. So, a physical consequence of this fact is that the speed of the Newtonian velocity that the instantaneous comoving observer $\partial_{t}(p)$ measures for the normal observer $N(p)$ does not approach the speed of light on $\Sigma^{n}$ (see [17], Sections 2.1 and 3.1, and [18]; see also [19], Chapter 12). 


\section{Hyperbolic Domains of $\mathbb{H}_{1}^{n+1}$}

When a compact spacelike hypersurface $\psi: \Sigma^{n} \rightarrow \mathbb{H}_{1}^{n+1}$ is entirely contained into some slice $\mathbb{H}_{t_{0}}^{n}=\left\{t_{0}\right\} \times \mathbb{H}^{n}$, it is called a hyperbolic domain of $\mathbb{H}_{1}^{n+1}$. As applications of Theorem 3.2, we obtain the following results.

Proposition 4.1. Let $\psi: \Sigma^{n} \rightarrow \mathbb{H}_{1}^{n+1}$ be a compact spacelike hypersurface whose boundary $\partial \Sigma$ is contained in some slice $\mathbb{H}_{t_{0}}^{n}$. Suppose that $\Sigma^{n}$ is not a hyperbolic domain, i.e. mean curvature $H>1$ is constant, and that one of the following conditions is satisfied.

(i) $-\pi / 2<t_{0} \leq 0$ and $\Sigma^{n}$ is contained into the chronological past with respect to $\mathbb{H}_{t_{0}}^{n}$.

(ii) $0 \leq t_{0}<\pi / 2$ and $\Sigma^{n}$ is contained into the chronological future with respect to $\mathbb{H}_{t_{0}}^{n}$.

Then

$$
H \leq 1+\frac{C \cos t_{0}-\cos h^{*}}{\left|h^{*}-t_{0}\right|}
$$

where $C=\max _{\partial \Sigma}(\cosh \theta), \theta$ is the hyperbolic angle between the Gauss map $N$ of $\Sigma$ and $\partial_{t}$, and $h^{*}=\max _{\Sigma} h$.

In what follows, we say that $\Sigma^{n}$ is tangent to $\mathbb{H}_{t_{0}}^{n}$ along its boundary $\partial \Sigma$ if $\partial \Sigma$ is contained into $\mathbb{H}_{t_{0}}^{n}$, and the restriction of the Gauss map $N$ of $\Sigma^{n}$ to $\partial \Sigma$ is equal to $\left(\partial_{t}\right)_{t=t_{0}}$ or $-\left(\partial_{t}\right)_{t=t_{0}}$ (that is, the hyperbolic angle between $N$ and $\partial_{t}$ is identically zero along $\partial \Sigma$ ).

Proposition 4.2. Let $\psi: \Sigma^{n} \rightarrow \mathbb{H}_{1}^{n+1}$ be a compact spacelike hypersurface, which is tangent to some slice $\mathbb{H}_{t_{0}}^{n}$ along its boundary. Suppose that $\Sigma^{n}$ is not a hyperbolic domain, that its mean curvature $H>1$ is constant and that one of the following conditions is satisfied.

(i) $-\pi / 2<t_{0} \leq 0$ and $\Sigma^{n}$ is contained into the chronological past with respect to $\mathbb{H}_{t_{0}}^{n}$.

(ii) $0 \leq t_{0}<\pi / 2$ and $\Sigma^{n}$ is contained into the chronological future with respect to $\mathbb{H}_{t_{0}}^{n}$.

Then

$$
1<H \leq 1+\left|\sin t_{0}\right|<2
$$

Proof. Initially, we observe, from Proposition 4.1 and from our assumption, that $\Sigma^{n}$ is tangent to $\mathbb{H}_{t_{0}}^{n}$ along its boundary:

$$
H \leq 1+\frac{\cos t_{0}-\cos h(p)}{\left|h(p)-t_{0}\right|}
$$

for all $p \in \Sigma^{n}$ such that $h(p) \neq t_{0}$. Therefore, taking in the previous inequality the limit $h(p) \rightarrow$ $t_{0}$, we conclude that

$$
H \leq 1+\left|\sin t_{0}\right|<2 \text {. }
$$


As a consequence of the previous result, we get the following characterization of hyperbolic domains of $\mathbb{H}_{1}^{n+1}$.

Corollary 4.3. Let $\psi: \Sigma^{n} \rightarrow \mathbb{H}_{1}^{n+1}$ be a compact spacelike hypersurface, which is tangent to some slice $\mathbb{H}_{t_{0}}^{n}$ along its boundary. Suppose that one of the following conditions is satisfied.

(i) $-\pi / 2<t_{0} \leq-\arctan 2$ and $\Sigma^{n}$ is contained into the chronological past with respect to $\mathbb{H}_{t_{0}}^{n}$.

(ii) $\arctan 2 \leq t_{0}<\pi / 2$ and $\Sigma^{n}$ is contained into the chronological future with respect to $\mathbb{H}_{t_{0}}^{n}$.

If its mean curvature $H \geq 2$ is constant, then $\Sigma^{n}$ is a hyperbolic domain.

Finally, we obtain the following nonexistence results.

Corollary 4.4. There is no compact spacelike hypersurface $\psi: \Sigma^{n} \rightarrow \mathbb{H}_{1}^{n+1}$ tangent to some slice $\mathbb{H}_{t_{0}}^{n}$ along its boundary, with constant mean curvature $H \geq 2$ and satisfying one of the following conditions.

(i) $\Sigma^{n}$ is contained into the chronological past with respect to $\mathbb{H}_{t_{0}}^{n}$, with- $\arctan 2<t_{0} \leq 0$.

(ii) $\Sigma^{n}$ is contained into the chronological future with respect to $\mathbb{H}_{t_{0}}^{n}$, with $0 \leq t_{0}<\arctan 2$.

Corollary 4.5. There is no compact spacelike hypersurface $\psi: \Sigma^{n} \rightarrow \mathbb{H}_{1}^{n+1}$ with constant mean curvature $H \geq 2$ and tangent to the slice $\mathbb{H}_{0}^{n}$ along its boundary.

\section{Acknowledgments}

The second author wants to thank Professor Antonio Gervasio Colares for his guidance. The first author is partially supported by FAPESQ/CNPq/PPP. The second author was partially supported by CAPES, Brazil. The authors wish to thank the referees for the useful and constructive suggestions and comments.

\section{References}

[1] J. Marsden and F. Tipler, "Maximal hypersurfaces and foliations of constant mean curvature in general relativity," Bulletin of the American Physical Society, vol. 23, p. 84, 1978.

[2] S. M. Stumbles, "Hypersurfaces of constant mean extrinsic curvature," Annals of Physics, vol. 133, no. 1, pp. 28-56, 1981.

[3] E. Calabi, "Examples of Bernstein problems for some nonlinear equations," Proceedings of Symposia in Pure Mathematics, vol. 15, pp. 223-230, 1970.

[4] S. Y. Cheng and S. T. Yau, "Maximal space-like hypersurfaces in the Lorentz-Minkowski spaces," Annals of Mathematics, vol. 104, no. 3, pp. 407-419, 1976.

[5] L. J. Alías and J. M. Malacarne, "Spacelike hypersurfaces with constant higher order mean curvature in Minkowski space-time," Journal of Geometry and Physics, vol. 41, no. 4, pp. 359-375, 2002.

[6] L. J. Alías and J. A. Pastor, "Constant mean curvature spacelike hypersurfaces with spherical boundary in the Lorentz-Minkowski space," Journal of Geometry and Physics, vol. 28, no. 1-2, pp. 85-93, 1998.

[7] L. J. Alías and J. A. Pastor, "Spacelike hypersurfaces with constant scalar curvature in the LorentzMinkowski space," Annals of Global Analysis and Geometry, vol. 18, no. 1, pp. 75-83, 2000.

[8] L. J. Alías, R. López, and J. A. Pastor, "Compact spacelike surfaces with constant mean curvature in the Lorentz-Minkowski 3-space," The Tohoku Mathematical Journal, vol. 50, no. 4, pp. 491-501, 1998.

[9] R. López, "Area monotonicity for spacelike surfaces with constant mean curvature," Journal of Geometry and Physics, vol. 52, no. 3, pp. 353-363, 2004. 
[10] D. Hoffman, J. H. S. de Lira, and H. Rosenberg, "Constant mean curvature surfaces in $M^{2} \times \mathbb{R}$," Transactions of the American Mathematical Society, vol. 358, no. 2, pp. 491-507, 2006.

[11] H. F. de Lima, "A sharp height estimate for compact spacelike hypersurfaces with constant $r$-mean curvature in the Lorentz-Minkowski space and application," Differential Geometry and Its Applications, vol. 26, no. 4, pp. 445-455, 2008.

[12] H. F. de Lima, "Spacelike hypersurfaces with constant higher order mean curvature in de Sitter space," Journal of Geometry and Physics, vol. 57, no. 3, pp. 967-975, 2007.

[13] A. G. Colares and H. F. de Lima, "Space-like hypersurfaces with positive constant $r$-mean curvature in Lorentzian product spaces," General Relativity and Gravitation, vol. 40, no. 10, pp. 2131-2147, 2008.

[14] A. Caminha and H. F. de Lima, "Complete spacelike hypersurfaces in conformally stationary Lorentz manifolds," General Relativity and Gravitation, vol. 41, no. 1, pp. 173-189, 2009.

[15] A. Caminha and H. F. de Lima, "Complete vertical graphs with constant mean curvature in semiRiemannian warped products," Bulletin of the Belgian Mathematical Society, vol. 16, no. 1, pp. 91-105, 2009.

[16] A. L. Albujer and L. J. Alías, "Spacelike hypersurfaces with constant mean curvature in the steady state space," Proceedings of the American Mathematical Society, vol. 137, no. 2, pp. 711-721, 2009.

[17] R. K. Sachs and H. H. Wu, General Relativity for Mathematicians, Graduate Texts in Mathematics, Springer, New York, NY, USA, 1977.

[18] J. M. Latorre and A. Romero, "Uniqueness of noncompact spacelike hypersurfaces of constant mean curvature in generalized Robertson-Walker spacetimes," Geometriae Dedicata, vol. 93, pp. 1-10, 2002.

[19] B. O'Neill, Semi-Riemannian geometry, vol. 103, Academic Press, London, UK, 1983.

[20] S. Carroll, Spacetime and Geometry: An Introduction to General Relativity, Addison Wesley, San Francisco, Calif, USA, 2004.

[21] R. M. Wald, General Relativity, University of Chicago Press, Chicago, Ill, USA, 1984.

[22] S. Weinberg, Gravitation and Cosmology: Principles and Applications of the General Theory of Relativity, John Wiley \& Sons, New York, NY, USA, 1972.

[23] L. J. Alías, A. Romero, and M. Sánchez, "Uniqueness of complete spacelike hypersurfaces of constant mean curvature in generalized Robertson-Walker spacetimes," General Relativity and Gravitation, vol. 27, no. 1, pp. 71-84, 1995.

[24] A. L. Besse, Einstein Manifolds, vol. 10 of Results in Mathematics and Related Areas (3), Springer, Berlin, Germany, 1987.

[25] S. Montiel, "Uniqueness of spacelike hypersurfaces of constant mean curvature in foliated spacetimes," Mathematische Annalen, vol. 314, no. 3, pp. 529-553, 1999.

[26] L. J. Alías, A. Brasil Jr., and A. G. Colares, "Integral formulae for spacelike hypersurfaces in conformally stationary spacetimes and applications," Proceedings of the Edinburgh Mathematical Society, vol. 46, no. 2, pp. 465-488, 2003.

[27] L. J. Alías and A. G. Colares, “Uniqueness of spacelike hypersurfaces with constant higher order mean curvature in generalized Robertson-Walker spacetimes," Mathematical Proceedings of the Cambridge Philosophical Society, vol. 143, no. 3, pp. 703-729, 2007.

[28] A. Barros, A. Brasil Jr., and A. Caminha, "Stability of spacelike hypersurfaces in foliated spacetimes," Differential Geometry and Its Applications, vol. 26, no. 4, pp. 357-365, 2008.

[29] S. W. Hawking and G. F. R. Ellis, The large Scale Structure of Space-Time, Cambridge University Press, Cambridge, UK, 1973. 


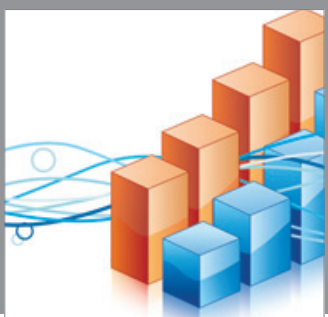

Advances in

Operations Research

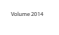

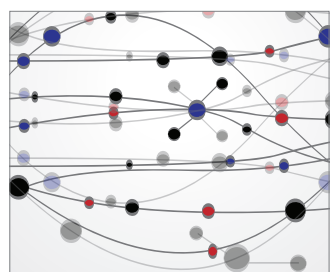

\section{The Scientific} World Journal
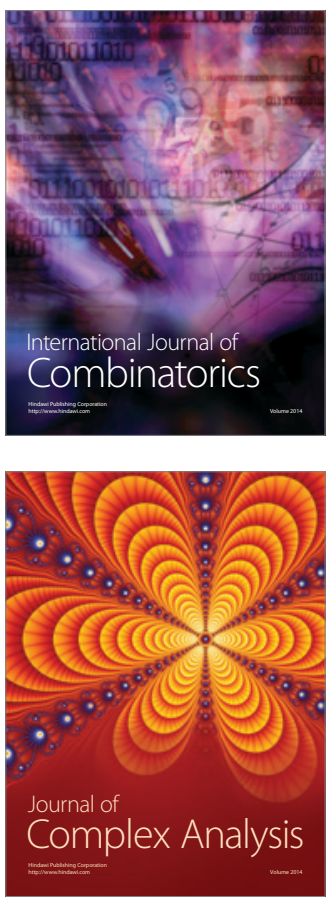

International Journal of

Mathematics and

Mathematical

Sciences
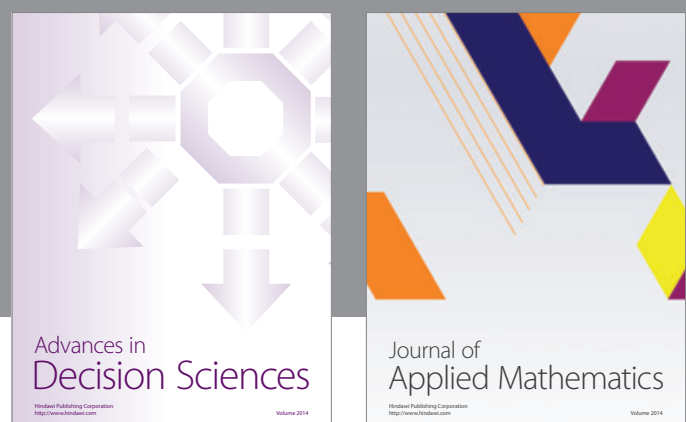

Journal of

Applied Mathematics
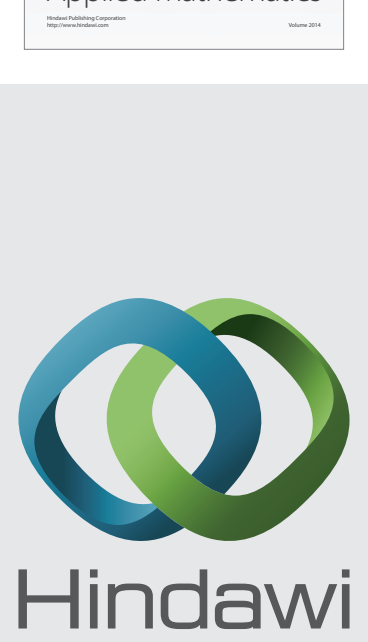

Submit your manuscripts at http://www.hindawi.com
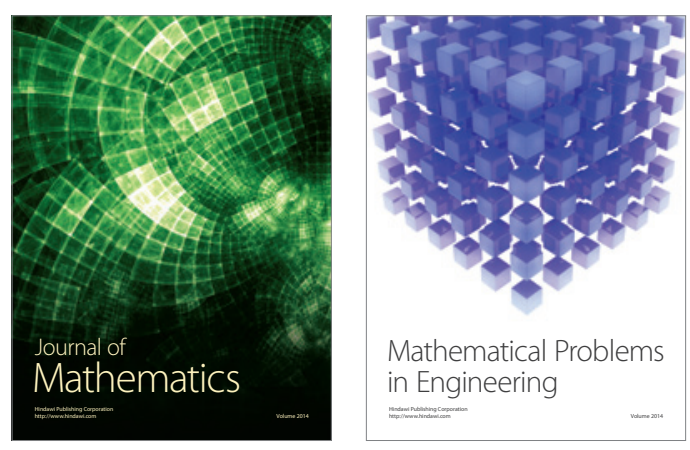

Mathematical Problems in Engineering
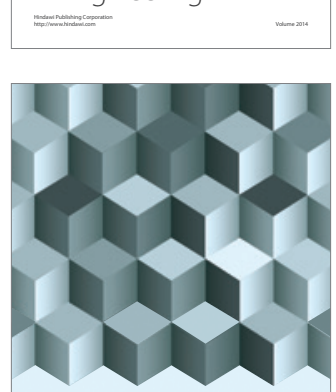

Journal of

Function Spaces
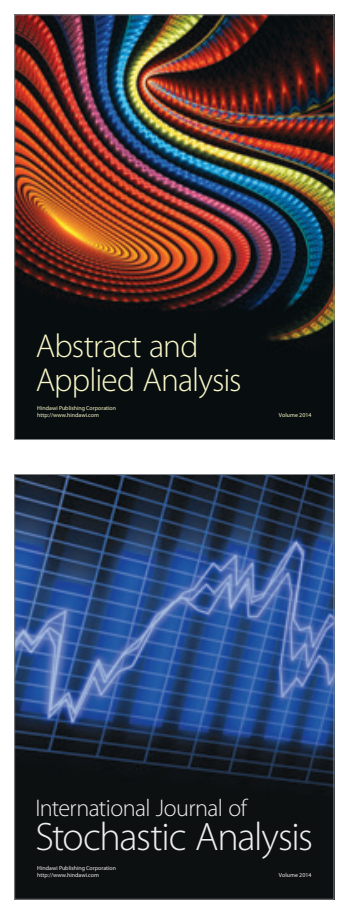

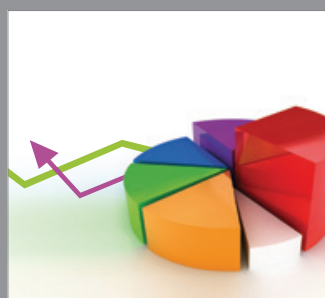

ournal of

Probability and Statistics

Promensencen
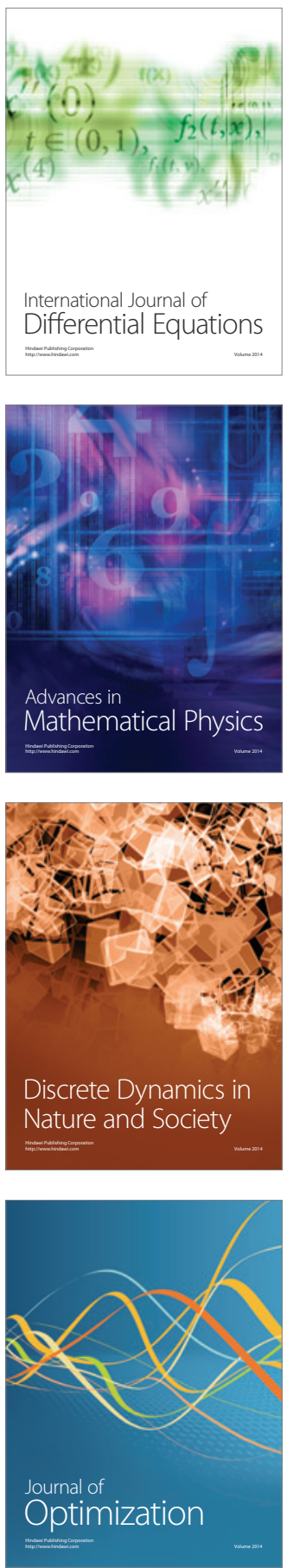